fournal of Medical Genetics (1972). 9, 57.

\title{
The Use of the AutoAnalyzer to Determine the Acetylator Phenotype
}

\author{
L. C. EZE and DAVID. A. PRICE EVANS
}

\section{From the Nuffield Unit of Medical Genetics, Department of Medicine, University of Liverpool, Liverpool L69 3BX}

It has been pointed out (Rao et al, 1970) that with the advent of intermittent treatment regimens the determination of the acetylator phenotype has become of practical importance. It has recently been shown that rapid acetylators have an inferior response to slow acetylators when their tuberculosis is treated with once weekly treatment regimens (Menon, 1968; Tuberculosis Chemotherapy Centre, Madras, 1970).

Sulfamethazine is polymorphically acetylated in man by the same $\mathrm{N}$-acetyl transferase as isoniazid (Evans and White, 1964).

The stable storage properties, easy analysis, and speedy availability of results have led to sulfamethazine acetylation being developed as a phenotyping test in preference to isoniazid (Evans and White, 1964; Evans, 1969; Rao et al, 1970).

It has been shown that a single small dose of $40 \mathrm{mg}$ sulfamethazine per $\mathrm{kg}$ body weight is sufficient (Evans, 1969); and either a single urine specimen (Rao et al, 1970), or alternatively one urine and one serum (or plasma) specimen (Evans, 1969) are required for analysis.

The purpose of the present communication is to show that a Technicon AutoAnalyzer can be used to perform the analyses required in the acetylator phenotyping procedure.

\section{Materials}

Sodium nitrite (British Drug Houses 'Analar' analytical reagent); ammonium sulphamate and $\mathrm{N}$-1-naphthylethylene-diamine dihydrochloride (British Drug Houses Laboratory reagents).

The Technicon AutoAnalyzer flow diagram is shown in Fig. 1 and the details of the modules and the solutions are given in the caption.

\section{Method}

Estimation of sulfamethazine concentration in urine and serum samples was performed by a modification of

Received 19 May 1971. the method of Falk and Kelly (1965), an automated adaptation of the Bratton-Marshall procedure for sulphonamides.

While serum samples generally require no dilution prior to analysis, some urine samples require dilution 1 in 10 with water, while others require no dilution.

The sampling module of the system was set to operate at a rate of 40 samples per hour, with a distilled waterwash between samples.

Free sulfamethazine was determined by first diluting the sample with $0.4 \mathrm{~N}$ hydrochloric acid. The acidification step before dialysis was incorporated in order to bring about the release of protein-bound sulfamethazine in serum or protein-containing urine samples (Falk and Kelly, 1965). The acidified sample was then dialysed into $0.3 \mathrm{~N}$ hydrochloric acid and the dialysate properly mixed by passing the sample line through a double-length mixing coil before diazotization. Excess nitrite after diazotization was removed by reacting with ammonium sulphamate before coupling with naphthylethylene-diamine dihydrochloride.

The sample stream was then passed through a single mixing coil immersed in water at $37^{\circ} \mathrm{C}$, mounted beneath the membrane dialysis module, and fed into a 15minute delay coil to allow full colour development. The stream was finally read at $540 \mathrm{~m} \mu$ and the absorbances recorded on a strip chart recorder.

For total sulfamethazine concentration, the mixed dialysate was passed into an $80 \mathrm{ft}$ long coil in a heating bath at $95^{\circ} \mathrm{C}$ for the hydrolysis of acetyl-sulfamethazine. (The changes of circuit are effected very easily by slipping the plastic transmission tubing off and on to plastic nipples.) The stream was then passed through a double-length mixing coil immersed in water at $20^{\circ} \mathrm{C}$ to reduce the temperature of the sample stream before diazotization. Thereafter, the stream was treated as for free sulfamethazine estimation.

An array of standard aqueous solutions of sulfamethazine at $30,60,90,120,150$, and $180 \mu \mathrm{g} / \mathrm{ml}$ concentration was run before and after each batch of unknowns.

\section{Results}

The results of phenotyping healthy students, doctors, nurses and laboratory staff with procedure II of Evans (1969) and performing the analyses on 


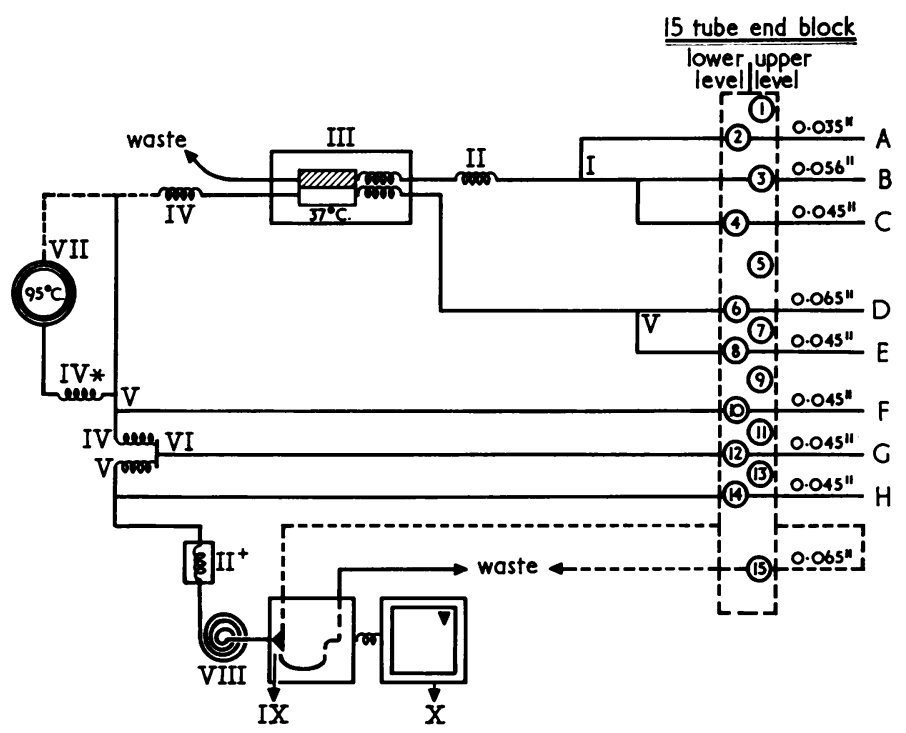

Fig. 1. Flow diagram for automated determination of free and total sulfamethazine in urine and serum (or plasma) using the $\stackrel{\widehat{S}}{\overrightarrow{3}}$ Technichon AutoAnalyzer.

Key to components: I-Cactus special with 0.023 I.D. plat. (Cat. no. H3). II-Mixing coil, standard size (Cat. no. MSC-1). II+ $\overrightarrow{0}$ Mixing coil, standard size (Cat. no. MSC-1) immersed in water with temperature controlled at $37^{\circ} \mathrm{C}$ (one of the additional coils mounted in the $37^{\circ} \mathrm{C}$-bath beneath the membrane dialysis module of III). III-Dialyzer, temperature controlled at $37^{\circ} \mathrm{C}$ (Cat. no. 105-A00-01) IV-Mixing coil, double length (Cat. no. MSC-II). IV*-Mixing coil, double length (Cat. no. MSC-II) immersed in water at $20^{\circ} \mathrm{C}^{*}$. V-h Junction (Cat. no. D1), VI-T junction, standard (Cat. no. A0). VII-Heating bath $\left(95^{\circ} \mathrm{C}\right)$, double coil $(1.6 \mathrm{~mm} \mathrm{I.D.} \times 80 \mathrm{ft})$ (Cat. no. 105-A100-01). VIII-Time delay coil (see text). IX-Flow cell, tubular $15 \mathrm{~mm}$ (N. method) (Cat. no. 112-728-15). $\mathbf{X}-$ Recorder, single pen. (Cat. no. 011-A00-01). A-Sample line. B-0.4N hydrochloric acid. C-Air. D-0.3N hydrochloric acid E-Air. F-0.1\% sodium nitrite. G-0.5\% ammonium sulphamate. $\mathbf{H}-0.1 \% \mathrm{~N}-(1-$ naphthyl $)$ ethylene diamine dihydrochloride.

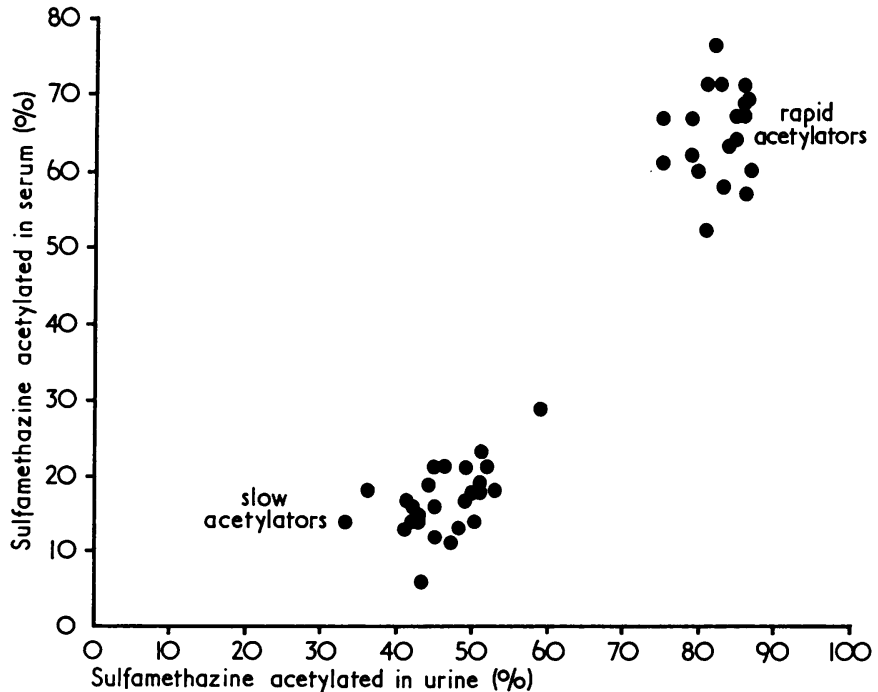

FIG. 2. Results of acetylator phenotype determinations performed using the apparatus shown in Fig. 1. 
the AutoAnalyzer, are shown in Fig. 2. The phenotypes are easily identified. When a scattergram was constructed from the results of analysing the same specimens manually, it looked closely similar to Fig. 2. Plasma samples give very similar results to serum samples by this AutoAnalyzer technique.

\section{Conclusion}

Technicon AutoAnalyzer apparatus can be used to determine 'free' (ie, unconjugated) and 'total' (ie, free plus acetylated) sulfamethazine concentrations in urine and serum (or plasma). Large numbers of subjects can be accurately acetylator-phenotyped by this technique, which may, therefore, be a contribution to the institution of more rational and successful therapy with 'intermittent' (eg, once weekly) dosage regimens for tuberculosis.

\section{Summary}

Technicon AutoAnalyzer modules can be utilized to estimate 'free' and 'total' sulfamethazine concentrations in urine and serum (or plasma). The laboratory procedures required for acetylator phenotyping of populations (for such purposes as monitoring of 'intermittent' tuberculosis chemotherapy or gene frequency determinations) can thus be automated.

The authors would like to thank the Medical Research Committee of United Liverpool Hospitals (Chairman, Professor C. A. Clarke, FRS) and the Nuffield Foundation (via Professor C. A. Clarke, FRS), also Mr Hasse Schröder of Uppsala, Sweden, who aided in obtaining all the serum samples.

\section{REFERENCES}

Evans, D. A. P. (1969). An improved and simplified method of detecting the acetyltor phenotype. fournal of Medical Genetics, 6 405-407.

Evans, D. A. P. and White, T. A. (1964). Human acetylation polymorphism. Fournal of Laboratory and Clinical Medicine, 63, 394-403.

Falk, H. B. and Kelly, R. G. (1965). An automated method for the determination of sulphonomides in plasma. Clinical Chemistry, 11, 1045-1050.

Menon, N. K. (1968). Madras study of supervised once-weekly chemotherapy. Bulletin of the International Union Against Tuberculosis, 41, 316-321.

Rao, K. V. N., Mitchison, D. A., Nair, N. G. K., Prema, K., and Tripathy, S. P. (1970). Sulphadimidine acetylation test for classification of patients as slow or rapid inactivators of Isoniazid. British Medical fournal, 3, 495-497.

Tuberculosis Chemotherapy Centre, Madras (1970). A controlled comparison of a twice-weekly and three once-weekly regimens in the initial treatment of pulmonary tuberculosis. Bulletin of the World Health Organisation, 43, 143-206. 